\title{
SenseBack - Implant considerations for an implantable neural stimulation and recording device
}

\author{
Ian Williams ${ }^{1}$, Adrien Rapeaux ${ }^{1}$, Jack Pearson ${ }^{1}$, Kianoush Nazarpour ${ }^{2}$, \\ Emma Brunton ${ }^{2}$, Song Luan ${ }^{1}$, Yan $\mathrm{Liu}^{1}$, and Timothy G. Constandinou ${ }^{1}$ \\ ${ }^{1}$ Dept. of Electrical \& Electronic Eng. and Centre for Bio-Inspired Tech., Imperial College London, UK \\ ${ }^{2}$ School of Engineering and Institute of Neuroscience, Newcastle University, UK \\ Email: i.williams10@imperial.ac.uk
}

\begin{abstract}
This paper describes a fully implantable and highly compact neural interface platform for chronic $(>6$ month) rat and small rodent experiments. It provides 32 channels of highly flexible neural stimulation and recording with wireless control and data readout, as well as wireless transcutaneous power. All the system firmware is fully upgradeable over the air (even after implantation) allowing future enhancements such as closed loop operation or data filtering. This paper focuses on the implant considerations - i.e. design and manufacture of the physical platform, encapsulation, wireless connections and testing.
\end{abstract}

\section{INTRODUCTION}

There are millions of amputees worldwide and thousands of people every year suffer major limb amputation. In the past 20 years there have been great leaps in prosthetic limb capability, however, even advanced prosthetics typically rely on feedforward control and limited automation. This lack of feedback is especially limiting for hand prosthetics where sensation is crucial for dextrous manipulation and tactile exploration.

The SenseBack project ${ }^{1}$ was founded to investigate neural interface technologies that could provide prosthetic limb users with artificial sensory feedback. The work focuses on proprioceptive and tactile feedback as these are believed to be of particular importance for enhancing prosthetic upper limb control, effectiveness and acceptability [1]. As part of SenseBack, an investigative platform (see Fig. 1) suitable for chronic implantation in a rat is required to enable the developed neural interface technology to be demonstrated and neuroscience experiments to be performed.

Previous implantable systems for animal neuroscience experiments consist of only a few channels of neural stimulation or recording and typically offer very limited control of stimulation parameters [2]-[5].Here we present the first 32 channel fully implantable neural stimulator and recording device suitable for rodent implantation. The device wirelessly communicates bidirectionally at $2 \mathrm{Mbps}$ in the $2.4 \mathrm{GHz}$ band and the recording and stimulating settings are highly configurable. The system is designed to be wirelessly powered through the skin and to be able to survive inside an animal for up to 6 months.

The heart of the implant is a custom bidirectional neural interface ASIC, simulations of which were previously described in [6]. However, of equal importance and difficulty has been the development and validation of supporting electronics,

${ }^{1}$ http://www.senseback.com

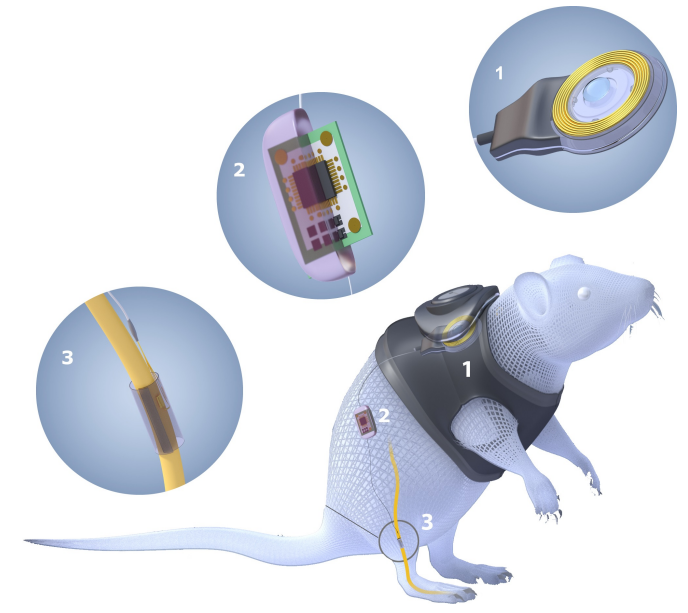

Fig. 1. Conceptual depiction of the SenseBack implant platform. (1) inductive, transcutaneous link between a backpack and implantable biotelemetry; (2) bidirectional neural interface implant; and (3) flexible, biocompatible penetrating microelectrode array implanted on a peripheral nerve.

firmware and physical system design for a chronic implanted electronic device. This paper describes our solutions to the the following key implant challenges:

- Wireless delivery of power;

- Power (and hence thermal) minimisation;

- Wireless communications with sufficient data;

- Fitting within tight space constraints and locations suitable for both neural interfacing and efficient transfer of wireless power and data;

- Packaging the device in a manner suitable for surgical implantation and 6 month implanted operation.

The remainder of this paper is organised as follows: Section II outlines the system architecture; Section III presents the detailed system design; Section IV presents the measured results; and Section V discusses the system performance and way forward.

\section{System ARChiteCtURE}

The top level system architecture (shown in Fig. 2) consists of 3 main parts: 1) a controller attached to a PC that wirelessly transmits stimulation commands and receives recorded data from the implant; 2) a fully implanted device which interfaces with the neural tissue; and 3) a battery backpack providing wireless transcutaneous power to the implant. 


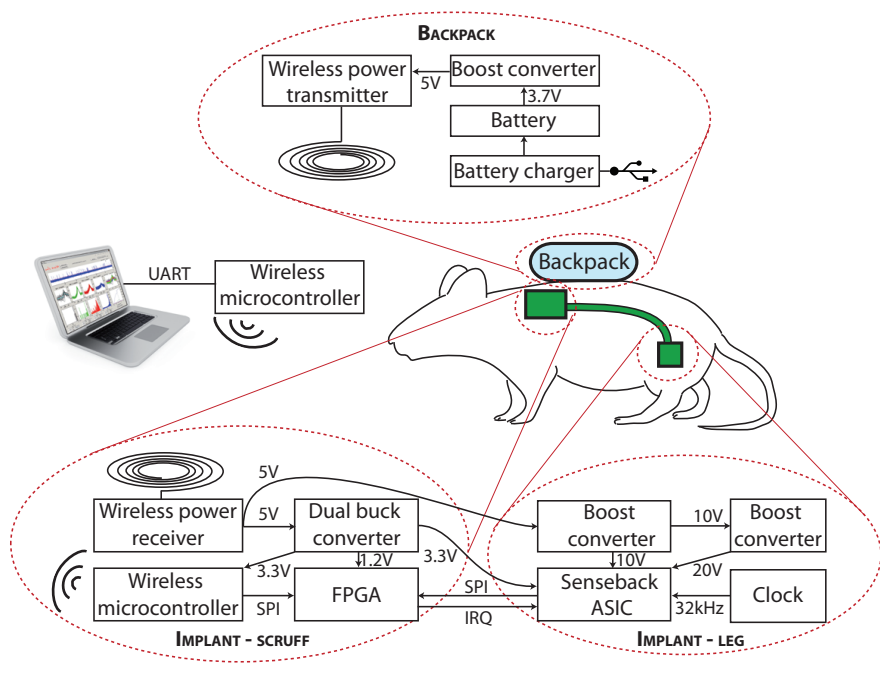

Fig. 2. The top level SenseBack system design.

The focus here is on the implant which has been designed to chronically stimulate and record from nerves and muscles in the hind leg of a large rat. The relatively long target implantation time (and associated need to minimise or ideally eliminate adverse effects on the rat) drove several key device decisions, most notably the purely wireless interface to avoid infections associated with skin penetrating wires or connectors. This in turn increases the required functionality (and hence size) of the implanted device as well as limiting placement sites. The implant must receive wireless power and stably generate all the required system voltage, receive and transmit wireless data and perform its key role of recording and stimulate neural and muscular tissue. 3 implant locations were considered (abdomen, neck scruff and hind leg), and based on space and surgical considerations a 2-part split implant was chosen, with wireless communications and power circuitry in the scruff of the neck and the neural interface electronics in the hind limb (see Figure 3).
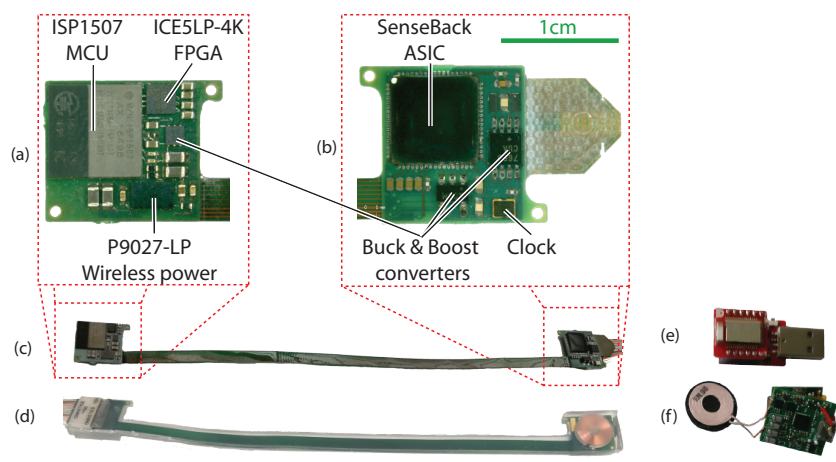

Fig. 3. The SenseBack system: (a) and (b) show closeups (to scale shown in (b)) of the scruff and leg ends of the implant respectively; (c) and (d) show the implant pre- and post-encapsulation respectively; (e) is the controller (a RedBear BLE nano v2) dongle; and (f) shows the backpack electronics.

\section{SySTEM DESIGN}

\section{A. System control and data flow}

There are numerous chips in the signal path between the controlling PC and SenseBack ASIC. Each of these chips passes data as transparently as possible - only grouping or separating the 16-bit data words as necessary for efficient transfer. The full signal path to the ASIC involves commands from the PC travelling through a virtual serial port to a microcontroller (MCU) development board and are wirelessly transmitted to the MCU in the implant. The implanted MCU passes the received data over SPI to a miniature Field Programmable Gate Array (FPGA) which acts as a high speed interface with the neural interface chip. Conversely, data from the chip comes through the FPGA, passes through the MCU and is wirelessly transmitted to the controller and PC.

\section{B. Neural interface}

The core of the implant is a custom neural interface ASIC (as described in [6] that utilises the same recording front end as described in [7]). The ASIC has 32 channels and is capable of high voltage neural stimulation and neural or ElectroMyoGraphic (EMG) recording on each of them. The IC is only $4 \times 4 \mathrm{~mm}$ but has been repackaged in a $9 \times 9 \mathrm{~mm}$ QFN package. Each neural recording channel is capable of being individually configured for neural recording and samples at $16 \mathrm{kHz}$ with 10-bit depth. The chip outputs a 16 bit data word for each sample (data and 5-bit channel identifier), and as such with all channels active in recording mode the chip outputs data at $\sim 8 \mathrm{Mbps}(32$ channels $\times 16$ bits $\times 16 \mathrm{kHz})$. To enable this data to be reliably communicated the chip operates as SPI master with a clock frequency of $16 \mathrm{MHz}$ and is equipped with an interrupt line to enable slave devices to request SPI communication. The chip additionally contains logic for neural spike detection and windowing - enabling just spike snippets to be transmitted, which could massively lower the data rate and make on-node spike sorting (and hence closed loop neural stimulation) readily achievable.

\section{Wireless communications}

The high data output rate of the system is challenging for wireless transmission. It could be handled by standard WiFi protocols, however, these present major power and latency (and hence data buffering) issues and as a result a Nordic Semiconductor proprietary protocol - Enhanced ShockBurst (ESB) - was chosen. This provides consistent latency and greatly reduced power, but limits the data rate to below 2 Mbps. This limitation can be mitigated by only activating recording on a subset of channels simultaneously as well as by utilising the spike windowing functionality. The Nordic NRF52832 chip was chosen for its low power operation and its availability in small package sizes. Here the ISP1507 System On Chip (an $8 \times 8 \mathrm{~mm}$ package with integrated MCU, antenna, crystal and power filtering) was utilised.

Due to the need to encapsulate the devices, but desire to maximise long term flexiblity, an Over The Air (OTA) firmware update bootloader was programmed on the MCU. The FPGA is also programmed by the MCU (from its flash memory) every time the system boots and hence the FPGA image is part of the MCU OTA firmware package. As such the whole system can be reprogrammed wirelessly even after encapsulation or even implantation. Indeed, to ensure that the system is always recoverable, the bootloader is configured to always look for a Bluetooth update for 10 seconds at start up. 


\section{Wireless power and power conversion}

Power consumption is a key consideration for thermal safety and delivering stable transcutaneous wireless power remains a significant challenge. This is especially true for freely behaving rodents due to implant size constraints and the animal movements. Here we equipped the rats with a backpack containing a Lithium-Polymer battery, battery charger, boost converter, wireless power transmitter (P9235A-R) and a power transfer coil. Inductive power transfer is carried out by aligning this coil with another implanted in the scruff of the neck. Ferrite sheets help improve efficiency and reduce alignment requirements. On the receiving end a wireless power receiver (P9027LP-R) rectifies and outputs $5 \mathrm{~V}$ which is DC buck converted to $3.3 \mathrm{~V}$ and $1.2 \mathrm{~V}$ for the MCU and FPGA, as well as boost converted to $10 \mathrm{~V}$ and $20 \mathrm{~V}$ for the neural interface chip.

\section{E. System assembly and packaging}

Size is a major constraint for devices targeting implantation in rats. Our approach uses splits the system in 2 parts - making use of small pockets of space in the scruff and leg. This allows the wireless power transfer to be located in the scruff, where it is well positioned to interface with a backpack, and allows the neural interface to be positioned as close as possible to the target nerve in the hind leg. Connectors are highly undesirable from a size and encapsulation perspective so a polyimide flexrigid PCB was chosen. The rigid elements at each end are where the electronics can be affixed (as well as suture points for fixing the implant in place) and the $\sim 100 \mu$ m thick flex ribbon can be tunneled under the skin between them.

Due to the size constraints and 2-part design, a non-hermetic encapsulation approach was targeted. A number of USP VI approved polymers were identified in the literature and paryleneC, epoxy (EPO-TEK 377) and silicones (NuSil MED-6215 and MED3-4213) were selected. Each encapsulant was degassed, applied to clean interdigitated test boards and placed in saline at $70^{\circ} \mathrm{C}$ (an accelerated aging test), while the impedance was measured periodically to monitor encapsulation efficacy. All the encapsulants worked for a short time, but the parylene and epoxy methods failed well short of the target lifetime (N.B. there was insufficient sample size and optimisation of encapsulant thickness and application to draw firm conclusions on encapsulant reliability). In contrast the silicones tested gave consistently reliable results and easily passed a 6 month equivalent accelerated aging test. The silicones also had the advantage that they were soft and flexible - enabling encapsulation of the whole flexible PCB and minimising mechanical damage to surrounding tissue. One downside of the silicones was their relatively high viscosity, which led to concerns about potential voids underneath the BGA and QFN packages. This could lead to liquid water condensing and causing shorts or acting as an initiation point for encapsulation failure. This issue was mitigated by careful degassing of the MED-6215 silicone and applying the silicone while under partial vacuum before curing it at normal pressure. Lacking a vacuum liquid dispenser an improvised method using just the microscopic amounts of air trapped in the silicone was used (see Figure 4)

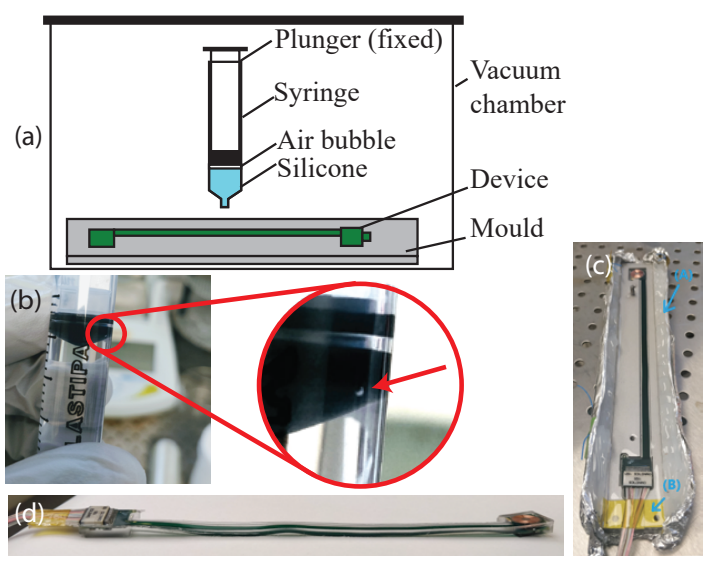

Fig. 4. Encapsulation of the implant: (a) partial vacuum silicone dispensing (b) example air bubble used for silicone ejection; (c) device mould; (d) side view of the encapsulated device, the silicone coating of the flexible section was hard to control but acceptably thick.

- the air bubble would expand as the air in the chamber was pumped out and expel the silicone.

\section{REsults}

\section{A. Neural interface}
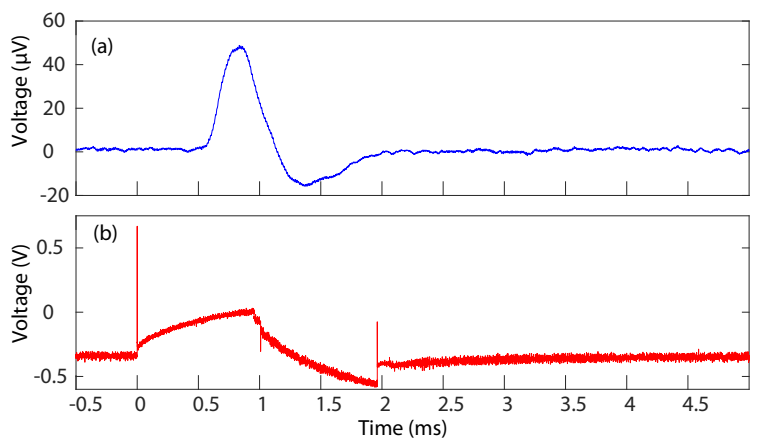

Fig. 5. Neural stimulation: (a) oscilloscope trace of an ex vivo compound action potential initiated by the SenseBack system; (b) the recorded electrode voltage of the SenseBack stimulation.

The stimulation circuitry has been fully tested in-silico and an opportunity was taken to test the device ex-vivo with a cuff electrode on approximately $4 \mathrm{~cm}$ of explanted rat sciatic nerve. The device successfully generated Compound Action Potentials as shown in Fig. 5.

The recording front end capability has been fully characterised and described in [7]. However, while the basic back end functionality has successfully been demonstrated the spike snippet output has not. We were also unable to take the opportunity to test the recording ex-vivo due to incompatibility with the system setup (grounding issues).

\section{B. Wireless data transmission}

It is well known that the $2.4 \mathrm{GHz}$ band is sub-optimal for implant data transmission. Benchtop testing with portions of meat (see Fig. 6) indicate that although there can be substantial attenuation, shallow implants (such as just below the skin of the neck scruff) should be viable. 

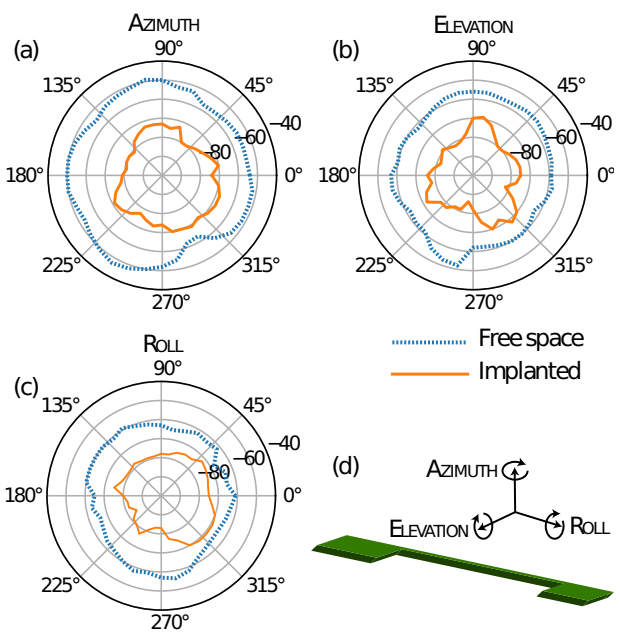

Free space

Implanted

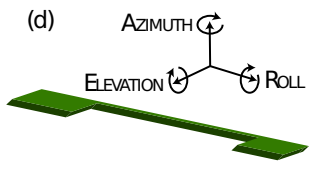

Fig. 6. Wireless signal strength at $1 \mathrm{~m}$ distance through free space (blue dashed lines) or implanted at $>5 \mathrm{~mm}$ depth in meat (orange solid lines) as device is rotated through (a) azimuth, (b) elevation and (c) roll. (d) indicates how each dimension relates to the device.
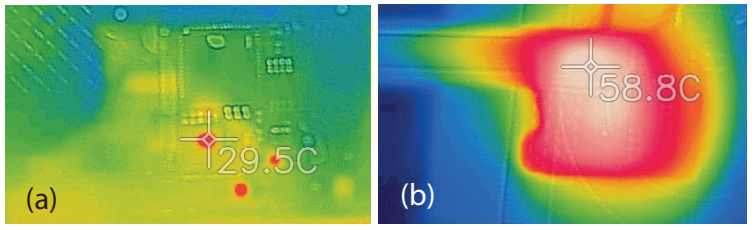

Fig. 7. Thermal images of: (a) the leg and (b) scruff end of the implant while wirelessly connected. The hotspot in (b) is the wireless power receiver.

\section{Wireless power}

The current wireless power transfer efficiency of the device is currently only around $20 \%$ with a significant portion of the remaining energy being dissipated as heat (both on the transmitter and receiver side of the link). This produces unacceptable levels of heating as shown in Fig. 7.

\section{System power}
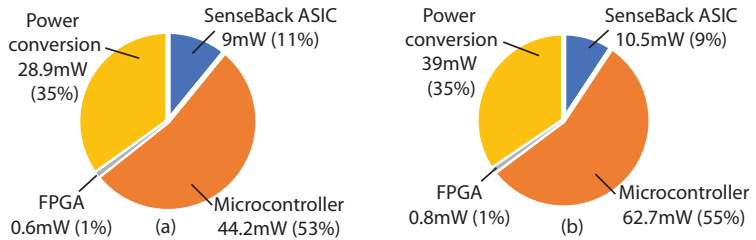

Fig. 8. System power breakdown: (a) under idle conditions $(83 \mathrm{~mW})$ and (b) while recording and wirelessly streaming data from 16 channels $(113 \mathrm{~mW})$. Wireless power efficiency not included

Fig. 8 shows the breakdown of the system power usage in both idle and recording modes. In both modes the power consumption is dominated by the microcontroller, as well as inefficiencies in the power conversion.

\section{E. Packaging}

Accelerated aging testing was performed on an encapsulated device (equipped with wire electrodes as in Fig. 4(d)) by placing it in a beaker filled with saline at $62^{\circ} \mathrm{C}$ and continuously powering it wirelessly through the glass. The device functioned throughout the 1 month test period, equivalent to approximately 6 months at $36^{\circ} \mathrm{C}$.

\section{DiscusSiON}

TABLE I

COMPARISON OF FULLY IMPLANTABLE RODENT NEURAL INTERFACES

\begin{tabular}{lllll}
\hline & $\begin{array}{l}\text { Shon } \\
\text { et al. } \text { [5] }\end{array}$ & $\begin{array}{l}\text { Paralikar } \\
\text { et al. } \text { [3] }\end{array}$ & $\begin{array}{l}\text { Xu } \\
\text { et al. } \text { [4] }\end{array}$ & $\begin{array}{l}\text { This } \\
\text { work }\end{array}$ \\
\hline \# record ch. & 2 & 0 & 0 & 32 \\
\# stim. ch. & 2 & 4 & 2 & 32 \\
Power source & $\begin{array}{l}\text { Inductive \& } \\
\text { secondary }\end{array}$ & $\begin{array}{l}\text { Inductive \& } \\
\text { secondary }\end{array}$ & Inductive Inductive \\
Packaging & $\begin{array}{l}\text { Photo- } \\
\text { polymer }\end{array}$ & Titanium & Silicone & Silicone \\
Volume $\left[\mathrm{cm}^{3}\right]$ & $\sim 11$ & $\sim 3$ & $\sim 3.5$ & $\sim 1.5$ \\
\hline
\end{tabular}

\section{A. Future work}

While demonstrating truly ground breaking performance for its size (see Table I) a number of issues and areas for further testing have been identified:

- Efforts are underway to improve wireless power transfer efficiency by better matching the impedance of the transmitter and receiver.

- Changes to the MCU firmware to reduce power consumption are being explored.

- Further testing of the recording backend and spike snippet windowing capability are being conducted.

- Implementation of spike sorting on the FPGA would be a significant bonus for brain based applications.

\section{B. Conclusion}

This paper described a 32 channel neural interface device that has been designed to be fully implanted in a rat. For its size this device offers almost an order of magnitude increase in the number of recording and stimulation channels compared to previous systems. The feasibility of key wireless data, power and encapsulation approaches has been investigated, as well as a novel encapsulated flex PCB approach to implant design.

\section{REFERENCES}

[1] A. Blank, A. M. Okamura, and K. J. Kuchenbecker, "Identifying the role of proprioception in upper-limb prosthesis control: Studies on targeted motion," ACM Transactions on Applied Perception (TAP), vol. 7, no. 3 , p. 15,2010 .

[2] R. E. Millard and R. K. Shepherd, "A fully implantable stimulator for use in small laboratory animals," Journal of neuroscience methods, vol. 166, no. 2, pp. 168-177, 2007.

[3] K. Paralikar et al., "A fully implantable and rechargeable neurostimulation system for animal research" in 2015 7th International IEEE/EMBS Conference on Neural Engineering (NER). IEEE, 2015, pp. 418-421.

[4] Q. Xu, D. Hu, B. Duan, and J. He, "A fully implantable stimulator with wireless power and data transmission for experimental investigation of epidural spinal cord stimulation," IEEE Transactions on Neural Systems and Rehabilitation Engineering, vol. 23, no. 4, pp. 683-692, 2015.

[5] A. Shon et al., "An implantable wireless neural interface system for simultaneous recording and stimulation of peripheral nerve with a single cuff electrode," Sensors, vol. 18, no. 1, p. 1, 2018.

[6] I. Williams et al., "A 32-ch. bidirectional neural/emg interface with onchip spike detection for sensorimotor feedback," in 2016 IEEE Biomedical Circuits and Systems Conference (BioCAS), Oct 2016, pp. 528-531.

[7] Y. Liu et al., "A 64-channel versatile neural recording soc with activitydependent data throughput," IEEE transactions on biomedical circuits and systems, vol. 11, no. 6, pp. 1344-1355, 2017. 